\title{
Evolution of Dissolution Media Over the Last Twenty Years
}

\author{
Jennifer Dressman \\ Institute of Pharmaceutical Technology, Goethe University, Frankfurt am Main, Germany
}

e-mail:dressman@em.uni-frankfurt.de

T wenty years ago, dissolution media were used almost exclusively for quality control purposes in the pharmaceutical industry. The most common dissolution tests were for oral dosage forms, and these were approached according to the release mechanismimmediate release (IR), enteric-coated (EC) tablets, and controlled-release (CR) tablets or pellets (beads).

In the USP from 1990 (1), water was quite commonly used as a dissolution medium. Alternatively, for poorly soluble drugs, solutions containing a surfactant, most commonly sodium lauryl sulfate or polysorbate 80 , were recommended to provide sink conditions. A third approach of the day was to use a medium that could reflect conditions in the stomach or small intestine. The medium for the stomach, Simulated Gastric Fluid Test Solution (SGF TS), was both acidic and contained pepsin, thus addressing two of the fundamental features of the composition of gastric fluids in the fasted state. Likewise, the medium for the small intestine, Simulated Intestinal Fluid Test Solution (SIF TS), was slightly basic (pH 7.5) and contained pancreatin to reflect the enzyme activity in this region.

For IR products, typically just one of the above media was recommended for dissolution testing. For example, theophylline IR products used water as a dissolution medium, metoprolol IR products used Simulated Gastric Fluid TS, and probenecid IR products used Simulated Intestinal Fluid TS. If a drug had pH-dependent solubility, a first line of approach was to use a buffer in which the drug had adequate solubility, as this made it easier to establish sink conditions. In some cases, the pH of SIF was not high enough to provide sink conditions, and for several products a more basic buffer was implemented (e.g., mephobarbital, for which a medium with a $\mathrm{pH}$ of 10.0 was used).

If there was no $\mathrm{pH}$ dependency in the solubility but the drug was poorly soluble, an aqueous solution of surfactant was typically used (e.g., carbamazepine). In some cases where the drug had $\mathrm{pH}$-dependent solubility but sink conditions could not be achieved in one of the buffer solutions, surfactant was added to the buffer with the best solubility (e.g., for spironolactone, the medium was $\mathrm{pH} 1.2$ plus sodium lauryl sulfate, SLS). Another approach for poorly soluble drugs was to add isopropyl alcohol to the medium, as in the case of dydrogesterone, or $n$-propyl alcohol as in the case of reserpine. Rarely, the volume of medium was increased to attain sink conditions, such as in the case of chloroxazone (1800 $\mathrm{mL})$.

These case examples underscore the emphasis on achieving sink conditions as a first priority, rather than try- ing to reflect how the drug product would perform under actual gastrointestinal (Gl) conditions.

A first step towards evoking a more physiological approach was the revision of the $\mathrm{pH}$ of simulated intestinal fluid from $\mathrm{pH} 7.5$ to $\mathrm{pH} 6.8$ (2), which came into force with USP 25. This change was made to reflect the fact that the $\mathrm{pH}$ in the small intestine typically reaches 7.5 toward the end of the ileum, whereas the IR dosage form or EC dosage form is expected to release the drug in the proximal small intestine to enable sufficient contact time between the drug and the intestinal mucosa for absorption.

By the time the USP General Chapter <1092> Dissolution Procedure: Development and Validation was introduced (3), it was recognized that physical and chemical data for the drug and dosage unit need to be determined before selecting the dissolution medium. Among other information, the solubility and solution state stability of the drug as a function of $\mathrm{pH}$ as well as, for less soluble drugs, the influence of surfactants on the solubility of the drug should be evaluated. For the dosage unit, the key factors are the intended release mechanism, the dosage range (if several strengths are to be produced), and any excipients that can modify the solubility properties of the drug.

While Chapter $<1092>$ continued to recommend that the dissolution medium be chosen to ensure that sink conditions are met, it was also noted that a medium that fails to provide sink conditions may be justifiable if it is shown to be more discriminating. The chapter also implied that the medium $\mathrm{pH}$ should not go outside the physiologic $\mathrm{pH}$ range of 1.2-7.5.

Chapter $<1092>$ also discouraged the use of an aqueous-organic solvent mixture as a dissolution medium, while remaining open to such a composition if an in vivoin vitro relationship had been demonstrated. Further, the pros and cons of water as a dissolution medium were extensively discussed, recognizing that while water is not representative of conditions in the Gl tract (physiological argument) and has no buffer capacity (reproducibility argument), it had been used for many drug products over a long period of time. All in all, with the introduction of this chapter in USP 30, we can see a shift away from "sink conditions at any price" to more consideration of what might be physiologically reasonable.

Revisions to Chapter $<1092>$ have recently been published in draft form $(4,5)$ and are under discussion as of this writing. With regard to the medium in which the dissolution test is conducted, the chapter continues to emphasize that a good knowledge of the solubility and 
Table 1. Composition of Various Gastric Biorelevant Media

\begin{tabular}{lccc}
\hline Component & SGF $_{\text {sp }}{ }^{a}$ & FaSSGF & FeSSGF \\
\hline Sodium taurocholate $(\mathrm{mM})$ & - & 0.08 & - \\
\hline Lecithin $(\mathrm{mM})$ & - & 0.02 & - \\
\hline Pepsin $(\mathrm{mg} / \mathrm{mL})$ & - & 0.1 & - \\
\hline Sodium chloride $(\mathrm{mM})$ & 34.2 & 34.2 & 237.0 \\
\hline Hydrochloric acid $(\mathrm{mM})$ & 71.0 & 25.1 & - \\
\hline Glacial acetic acid $(\mathrm{mM})$ & - & - & 17.1 \\
\hline Sodium acetate $(\mathrm{mM})$ & - & - & 29.8 \\
\hline Milk/Buffer & - & - & $1 / 1$ \\
\hline Characteristic parameter & & & \\
\hline pH & 1.2 & 1.6 & 5.0 \\
\hline Osmolality $(\mathrm{mOsm} / \mathrm{kg})$ & - & 120.7 & 400 \\
\hline Buffer capacity $(\mathrm{mmol} / \mathrm{L} / \Delta \mathrm{pH})$ & - & - & 25 \\
\hline
\end{tabular}

a Simulated gastric fluid without pepsin is included as a compendial medium for comparison.

stability of the drug substance in various media is key to selecting an appropriate dissolution medium. Surfactants may be added to the media to assist in attaining sink conditions for poorly soluble drug substances, in which case the dosage form effects on dissolution can be evaluated largely independently of the drug substance effects. However, the concentration of surfactant must be justified by comparing results obtained with media prepared at higher and lower concentrations of surfactant. As an alternative to the common synthetic surfactants, natural surfactants such as bile salts and lecithin can be used in the composition of media, which enables the dissolution method to more closely resemble conditions in the proximal human gastrointestinal tract.

In parallel to the evolution in expectations for quality control dissolution methods, the need to be able to forecast the in vivo performance of a drug during drug development has also been increasingly recognized. For this purpose, it is particularly important for poorly soluble drugs that the in vitro test mimic the conditions in vivo as closely as possible. To achieve this goal, biorelevant gastrointestinal media that simulate the fasted and fed states were first proposed in 1998 (6). These media, also discussed in the revisions to Chapter <1092> as indicated above, have been used to examine the solubility and dissolution characteristics of several classes of drugs (e.g., poorly soluble weak bases and weak acids, lipophilic drugs) to assist in predicting their in vivo absorption behavior (7-9).

Biorelevant in vitro dissolution testing is useful for qualitative forecasts of formulation and food effects on the dissolution and availability of orally administered drugs as well as for understanding how the drug product will release the drug in vivo. It has been observed that results in biorelevant media, when coupled with physiologically based pharmacokinetic models, can provide a more accu- rate simulation of pharmacokinetic profiles than results in the compendial simulated gastric and intestinal fluids (10).

Thus, the use of biorelevant media can greatly assist with the selection of drug product prototypes to optimize in vivo performance and to understand which dosing conditions are most appropriate. Also, many post-approval formulation changes (including new generic formulations) could be screened for bioequivalence using biorelevant dissolution testing prior to being entered into pharmacokinetic bioequivalence studies. As such, the biorelevant testing approach provides a means of reducing the risks associated with product changes and in designing generic versions of existing drug products.

The formulations and the instructions on how to prepare the biorelevant media originally developed by Dressman et al. can be found in a paper published in Dissolution Technologies in 2004 (11).

Since then, several updates and alternative media have been proposed. The compositions of the most widely used biorelevant media, FaSSGF, FaSSIF, FaSSIF-V2 and FeSSIF-V2, are provided in Tables 1 and 2. Additionally, a medium to reflect conditions in the stomach after meal intake, FeSSGF, has been published $(12,13)$. As this medium contains heat-treated milk as one of the ingredients, some concerns have been raised about possible variability in composition of the medium according to the source of the milk, the season, and so forth. Additionally, sample analysis is made difficult when working with this medium because of the need to separate the dissolved drug from the milk proteins. For this reason, one has to precipitate the proteins and then use rather coarse filters $(2.7-\mu \mathrm{m}$ pore size), which of course carries the risk that some fine, undissolved drug might also be present in the filtrate and thus contribute to the total concentration measured. For these reasons, efforts are underway to establish good alternatives to the milk-based medium that are easier to work with from an analytical point of view (14).

A question that is often raised during the formulation development phase is whether it is necessary to use biorelevant media to assist in comparison of prototype performance and to predict whether the drug-formulation combination will have a food effect. This question can often be answered in the framework of the Biopharmaceutics Classification Scheme (BCS). According to this scheme, which was first proposed by Amidon et al. in 1995 (15), each and every drug can be classified according to its solubility and intestinal permeability characteristics. As such, it applies exclusively to dosing drugs orally. If the drug should act systemically, both the permeability and the solubility classifications are important to screening for the ability of the drug to be absorbed, but if the drug should act locally in the gastrointestinal tract, the solubility may be the primary factor in assuring that the drug meets its target.

For drugs that are highly soluble according to the BCS, the use of simple aqueous buffers is recommended for screen- 
Table 2. Composition of the Biorelevant Small Intestinal Media

\begin{tabular}{|c|c|c|c|c|c|}
\hline Component (mM) & $\operatorname{SIF}_{\mathbf{s p}}{ }^{a}$ & FaSSIF-V2 & FaSSIF & FeSSIF-V2 & FeSSIF \\
\hline Sodium taurocholate & - & 3 & 3 & 10 & 15 \\
\hline Lecithin & - & 0.2 & 0.75 & 2 & 3.75 \\
\hline Glycerol monooleate & - & - & - & 5 & - \\
\hline Sodium oleate & - & - & - & 0.8 & - \\
\hline Maleic acid & - & 19.12 & - & 55.02 & - \\
\hline Monobasic sodium phosphate & - & - & 28.36 & - & - \\
\hline Monobasic potassium phosphate & 49.97 & - & - & - & - \\
\hline Glacial acetic acid & - & - & - & - & 144.05 \\
\hline Sodium hydroxide & 45 & 34.80 & 8.7 & 81.65 & 101 \\
\hline Sodium chloride & - & 68.62 & 105.85 & 125.5 & 203.18 \\
\hline \multicolumn{6}{|l|}{ Characteristic parameter } \\
\hline $\mathrm{pH}$ & 6.8 & 6.5 & 6.5 & 5.8 & 5.0 \\
\hline Osmolality (mOsm/kg) & - & $180 \pm 10$ & $270 \pm 10$ & $390 \pm 10$ & $670 \pm 10$ \\
\hline Buffer capacity (mmol/L/ pH) & - & $10 \pm 2$ & $10 \pm 2$ & $25 \pm 2$ & $76 \pm 2$ \\
\hline
\end{tabular}

a Simulated intestinal fluid without pancreatin is included as a compendial medium for comparison.

ing formulations. However, if the drug does not meet the criteria for highly soluble according to the BCS, it may be advisable to use biorelevant media to screen for formulation performance. Recently, a supplementary scheme (16), the Development Classification Scheme (DCS), has been developed to help the formulator more precisely assess drug characteristics within the framework of formulation development. This scheme is set up to assess whether drug dissolution is limited by particle size or by solubility, and thus gives the formulator valuable information not only about whether drug absorption will be limited by luminal events but also on which formulation strategies are most likely to succeed for the given drug to overcome these limitations.

In any case, whenever the solubility or dissolution is a limitation to drug absorption, biorelevant media are useful to assess formulation performance and food effects. This includes Class II and IV drugs of the BCS and Class Ila, Ilb, and IV drugs of the DCS, thus covering most drug candidates currently in the research and development pipeline of major innovator drug companies as well many of the drugs for which generic products are being developed.

With such a wide range of applications for biorelevant media, many companies are looking for ways of minimizing the costs and amount of time required to prepare the media. As one can imagine, with such a detailed composition, the biorelevant media take time and care to prepare in a reproducible manner. To make life easier for laboratory personnel and at the same time improve reproducibility of the media, instant versions of FaSSIF and FeSSIF have been introduced to the market by Biorelevant.com (www. biorelevant.com). The so-called "SIF powder" offered by this supplier is simply mixed with an already prepared buffer and warmed to the experimental temperature; it is then ready for the dissolution or release test (17).

One final issue that has been discussed extensively in the last years is that of the buffer choice for dissolution media. Buffers are important to ensure that the $\mathrm{pH}$ is maintained throughout the dissolution test and hence to support reproducibility of the results. This is of course primarily a consideration when the drug substance or the excipients in the formulation have $\mathrm{pH}$-dependent solubility or disintegration characteristics. While Chapter $<1092>$ focuses on dissolution for quality control purposes and suggests robust buffer systems like phosphates or acetates, some authors have suggested that since bicarbonate is the more prevalent buffer secreted in the intestines, it should be used for testing under intestinal conditions. The instability of the $\mathrm{pH}$ of bicarbonate buffers due to interaction with carbon dioxide from the atmosphere appears to be the biggest block to its use in vitro (18). However, efforts are underway to circumvent these issues for bicarbonate buffers (19).

\section{WHAT DOES THE FUTURE HOLD FOR DISSOLUTION MEDIA?}

As mentioned above and as reflected in the development of Chapter <1092> over the years, dissolution testing has been and will continue to be a paradigm in which the ability of the product to release the drug under physiologically relevant conditions is increasingly emphasized. Gone are the days of extreme $\mathrm{pH}$ values in media, huge media volumes, and organic solvent systems. This is because we increasingly recognize that quality in vitro should be linked to quality in vivo; that is, the drug product not only has to release the drug but also release it in a 
timely manner consistent with the therapeutic aims of the drug product. This new paradigm for dissolution testing is completely consistent with the tenets of Quality by Design and patient-centric drug development.

As we increase our level of knowledge of the physiology at the site of application, we will be able to design more appropriate tests for drug products, including refinement of the existing biorelevant media. As suggested recently, a set of media that takes into account the variability in human gastrointestinal physiology might be particularly helpful in predicting variations in dissolution and hence absorption, particularly for products containing poorly soluble drug substances (19). Clearly, to better represent conditions under which extended-release or pulsatilerelease drug products release the drug substances, more knowledge will have to be gained concerning the physiology in the lower gastrointestinal tract.

Further, for some drug substances, particularly those with good aqueous solubility (BCS Class I and III), and for those drug products with robust release patterns under gastrointestinal conditions (e.g., osmotic pump-based release), the use of simpler test designs is often warranted. So there is a clear need to establish some kind of decision tree to assist formulators and analysts working with dissolution to select the right level of biorelevance in the test design. In some cases, a simple buffer medium may well be adequate, while in other cases a more comprehensive simulation of gastrointestinal conditions will be appropriate. In these cases, we will look to instant versions of the biorelevant media to ensure reproducibility, cut costs, and reduce preparation time in the laboratory.

By coupling the results of dissolution testing with physiologically based pharmacokinetic modeling, we will be able to translate dissolution test results into plasma profiles, which in turn can lead the way to smart selection of formulations and the ability to recognize and mitigate food effects earlier in the development process. With this approach, we can provide the link between the laboratory and the patient that is crucial to the concept of Quality by Design and will ensure batch-to-batch and product-toproduct therapeutic equivalence. This will boost confidence in quality control dissolution testing on the part of industrial and regulatory scientists alike.

\section{REFERENCES}

1. The United States Pharmacopeia and National Formulary USP 22-NF 17; The United States Pharmacopeial Convention, Inc.: Rockville, MD, 1989.

2. Gray, V.; Dressman, J. B. Change of pH Requirements for Simulated Intestinal Fluid TS. Pharm. Forum 1996, 22 (1), 1943-1945.

3. Gray, V. A.; Brown, C. K.; Dressman, J. B.; Leeson, L. J. A New General Information Chapter on Dissolution. Pharm. Forum 2002, 27 (6), 3431-3439.

4. Skwierczynski, R.; Curry, P.; Gray, V.; Krämer, J.; Stippler, E.; Suggett, J.; Mirza, T.; Brown, W. Revision of The
Dissolution Procedure: Development and Validation $<1092>$. Pharm. Forum [Online] 2014, 40 (1).

5. Skwierczynski, R.; Curry, P.; Gray, V.; Krämer, J.; Stippler, E.; Suggett, J.; Mirza, T.; Brown, W. Revision of The Dissolution Procedure: Development and Validation $<1092>$. Dissolution Technol. 2014, 21 (1), 6-8.

6. Dressman, J. B.; Amidon, G. L.; Reppas, C.; Shah, V.P. Dissolution Testing as a Prognostic Tool for Oral Drug Absorption: Immediate Release Dosage Forms. Pharm. Res. 1998, 15 (1), 11-22. DOI: 10.1023/A:1011984216775.

7. Nicolaides, E.; Symillides, M.; Dressman, J. B.; Reppas, C. Biorelevant Dissolution Testing to Predict the Plasma Profile of Lipophilic Drugs After Oral Administration. Pharm. Res. 2001, 18 (3), 380-388. DOI: 10.1023/A:1011071401306.

8. Löbenberg, R.; Krämer, J.; Shah, V. P.; Amidon, G. L.; Dressman, J. B. Dissolution Testing as a Prognostic Tool for Oral Drug Absorption: Dissolution Behavior of Glibenclamide. Pharm. Res. 2000, 17 (4), 439-444. DOI: $10.1023 / A: 1007529020774$.

9. Kostewicz, E. S.; Brauns, U.; Becker, R.; Dressman, J. B. Forecasting the Oral Absorption Behavior of Poorly Soluble Weak Bases Using Solubility and Dissolution Studies in Biorelevant Media. Pharm. Res. 2002, 19 (3), 345-349. DOI: 10.1023/A:1014407421366.

10. Otsuka, K.; Shono, Y.; Dressman, J. Coupling biorelevant dissolution methods with physiologically based pharmacokinetic modelling to forecast in-vivo performance of solid oral dosage forms. J. Pharm. Pharmacol. 2013, 65 (7), 937-952. DOI: 10.1111/jphp.12059.

11. Marques, M. Dissolution Media Simulating Fasted and Fed States. Dissolution Technol. 2004, 11 (2), 16.

12. Jantratid, E.; Janßen, N.; Reppas, C.; Dressman, J. Dissolution Media Simulating Conditions in the Proximal Human Gastrointestinal Tract: An Update. Pharm. Res. 2008, 25 (7), 1663-1676. DOI: 10.1007/ s11095-008-9569-4.

13. Jantratid, E.; Dressman, J. Biorelevant Dissolution Media Simulating the Proximal Human Gastrointestinal Tract: An Update. Dissolution Technol. 2009, 16 (3), 21-25.

14. Kilic, M.; Arndt, M.; Jünemann, D.; Dressman, J. Fed State Simulated Gastric Emulsions-Biorelevant Dissolution Media Based on the Gastric Snapshot Approach. AAPS Annual Meeting \& Exposition, New Orleans, LA, Nov 15-17, 2010.

15. Amidon, G. L.; Lennernäs, H.; Shah, V. P.; Crison, J. R. A Theoretical Basis for a Biopharmaceutic Drug Classification: The Correlation of in Vitro Drug Product Dissolution and in Vivo Bioavailability. Pharm. Res. 1995, 12 (3), 413-420. DOI: 10.1023/A:1016212804288.

16. Butler, J. M.; Dressman, J. B. The developability classification system: Application of biopharmaceutics concepts to formulation development. J. Pharm. Sci. 2010, 99 (12), 4940-4954. DOI: 10.1002/jps.22217. 
17. Boni, J. E.; Brickl, R. S.; Dressman, J.; Pfefferle, M. L. Instant FaSSIF and FeSSIF-Biorelevance Meets Practicality. Dissolution Technol. 2009, 16 (3), 41-45.

18. Boni, J. E.; Brickl, R. S.; Dressman, J. Is bicarbonate buffer suitable as a dissolution medium? J. Pharm. Pharmacol. 2007, 59 (10), 1375-1382. DOI: 10.1211/jpp.59.10.0007.

19. Bergström, C. A.; Holm, R.; Jørgensen, S. A.; Andersson, S. B.; Artursson, P.; Beato, S.; Borde, A.; Box, K.; Brewster,
M.; Dressman, J.; Feng, K. I.; Halbert, G.; Kostewicz, E.; McAllister, M.; Muenster, U.; Thinnes, J.; Taylor, R.; Mullertz, A. Early pharmaceutical profiling to predict oral drug absorption: Current status and unmet needs. Eur. J. Pharm. Sci. 2013, 57 (16), 173-199. DOI: 10.1016/j.ejps.2013.10.015. 